\title{
Machine-Learning QSAR Model for Predicting Activity against Malaria Parasite's Ion Pump PfATP4 and In Silico Binding Assay Validation +
}

\author{
Angela Lopez-Del Rio 1,2, Laura Llorach-Parés ${ }^{1,3}$, Alexandre Perera-Lluna ${ }^{2,4}$, Conxita Avila ${ }^{3}$, \\ Alfons Nonell-Canals ${ }^{1}$ and Melchor Sanchez-Martinez ${ }^{1, *}$ \\ 1 Mind the Byte S.L., 08028 Barcelona, Spain; angela@mindthebyte.com (A.L.-D.R.); \\ laura@mindthebyte.com (L.L.-P.); alfons@mindthebyte.com (A.N.-C.) \\ 2 Department of ESAII, Center for Biomedical Engineering Research, Universitat Politècnica de Catalunya \\ (UPC), 08034 Barcelona, Spain; alexandre.perera@upc.edu \\ 3 Department of Evolutionary Biology, Ecology, and Environmental Sciences, Faculty of Biology and \\ Biodiversity Research Institute (IrBIO), Universitat de Barcelona, 08028 Barcelona, Spain; \\ conxita.avila@ub.edu \\ 4 Biomedical Research Networking Center in Bioengineering, Biomaterials and Nanomedicine (CIBER-BBN), \\ 28029 Madrid, Spain \\ * Correspondence: melchor@mindhtbeyte.com \\ + Presented at the 1st Molecules Medicinal Chemistry Symposium, Barcelona, Spain, 8 September 2017.
}

Published: 18 October 2017

Malaria is a mosquito-borne infectious disease caused by parasitic protozoans of the genus Plasmodium. Although different effective antimalarial medicines have been developed, there is serious concern that parasites are developing widespread resistance to these drugs. To avoid this, now the efforts are concentrated on treating the malaria inside the Anopheles mosquito.

Several academic groups and companies are working worldwide to develop new compounds that fight the disease without provoking drug resistance. Within this context and making use of the Open Source Malaria project (http://opensourcemalaria.org/) which provides a large collection of molecules and 3D models of the targets with which they interact, we developed a machine learningbased QSAR model that predicts which molecules will block the malaria parasite's ion pump, PfATP4. The model was then employed to screen and classify the DrugBank database molecules and compounds coming from a proprietary marine molecules library. Finally, by means of an in silico binding assay, the predicted behavior was validated in the positive cases.

Summarizing, we have created a new set of repositioned drugs and marine molecules against malaria, establishing a good starting point for further studies and highlighting the key role that computational methods can have in the rational design of new drugs against infectious diseases.

Acknowledgments: L.L-P and A.L.-D.R. acknowledge the Doctorats Industrials pre-doctoral grants 2016 DI 051 and 2016 DI 080, respectively, from the Generalitat de Catalunya.

Author Contributions: A.L-D.R. constructed the machine learning model and realized the QSAR experiments. L.L-P conducted the in silico binding experiments. A.P-L supervised the model generation, A.P-L and M.S-M supervised the QSAR experiments, A.N-C, C.A and M.S-M supervised the in silico binding experiments. All the authors analyzed and discussed the results. A.P-L and M.S-M conceived the study and designed the experiments. A.L-D.R and L-L.P wrote the abstract whereas A-N.C, C.A, A-P.L and M.S-M corrected it.

Conflicts of Interest: 'The authors declare no conflicts of interest'.

(C) 2017 by the authors. Licensee MDPI, Basel, Switzerland. This article is an open access article distributed under the terms and conditions of the Creative Commons Attribution (CC BY) license (http://creativecommons.org/licenses/by/4.0/). 